\title{
PRÁTICA SUSTENTÁVEL NA APS - EDUCAÇÃO E PATERNALISMO LIBERTÁRIO EM TEMA DE SAÚDE PÚBLICA
}

\author{
SUSTAINABLE PRACTICE IN PHC - EDUCATION AND LIBERTARIAN \\ PATERNALISM IN PUBLIC HEALTH
}

\author{
Henrique Ribeiro Cardoso ${ }^{1}$ \\ Fernando Gustavo Knoerr ${ }^{2}$ \\ Tatiana Moreira Afonso ${ }^{3}$
}

\section{RESUMO}

A Atenção Primária a Saúde (APS) é responsável por ações de saúde, no âmbito individual e coletivo, que abarca promoção, proteção da saúde, prevenção de agravos, estabelecimento de diagnóstico, tratamento, reabilitação, redução de danos e a manutenção da saúde, a fim de proporcionar uma atenção integral ao usuário do Sistema Único de Saúde, com a finalidade de surtir um efeito impactante nas condições de saúde da população, causando influencia na autonomia das pessoas e nos fatores determinantes do processo saúde doença da população. O emprego da educação em saúde pelas Equipes de Saúde da Família (eSF) tem sido de bastante valia, por ser um campo com várias peculiaridades que contribui para o desenvolvimento do indivíduo que tem como base a concepção de educação o que possibilita estímulo da reflexão, desenvolve consciência crítica, exerce autonomia, oferece possibilidade de transformar sua realidade e escrever a própria história. Nas políticas públicas de saúde podemos considerar o uso do Nudge para o impulsionamento de tomada de decisões individuais e coletivas para práticas saudáveis e sustentáveis. No caso do "paternalismo libertário",

\footnotetext{
${ }^{1}$ Doutor em Direito, Estado e Cidadania (UGF/Rio), com Pós-doutorado em Democracia e Direitos Humanos (IGC - Universidade de Coimbra) e Pós-doutorado em Direitos Humanos e Desenvolvimento (PPGCJ/UFPB); Mestre em Direito, Estado e Cidadania (UGF/Rio); Especialista em Direito Constitucional Processual (FAPESE/UFS); Graduado em Direito pela Universidade Estadual de Santa Cruz (UESC/Bahia); Professor do Programa de Pós-graduação da Universidade Federal de Sergipe (Mestrado/PRODIR/UFS); Professor de Programa de Pósgraduação da Universidade Tiradentes (Mestrado/PPGD/UNIT); Professor da Escola Superior do Ministério Público de Sergipe (ESMP/SE); Membro da Academia Sergipana de Letras Jurídicas (ASLJ/SE); Promotor de Justiça em Sergipe.

2 Doutor em Direito do Estado pela Universidade Federal do Paraná (2002). Mestre em Direito do Estado pela Universidade Federal do Paraná (1998). Bacharel em Direito pela Universidade Federal do Paraná (1993). Ex-Procurador Federal. Professor de Direito Administrativo da Escola da Magistratura do Paraná e da Fundação Escola do Ministério Público do Paraná / FEMPAR. É Professor Permanente do Programa de Mestrado em Direito Empresarial e Cidadania do UNICURITIBA.

${ }^{3}$ Possui graduação em Enfermagem e Obstetrícia pelo Centro de Estudos Superiores de Londrina/ PR - Cesulon (1997). Especialização em Saúde Pública (FAFIPA) (2001) e em Saúde da Família (UFMA) (2003), onde durante essa especialização foi bolsista do CNPq no Programa de Interiorização do Trabalho em Saúde - PITS, por três anos, atuando nos estados do Maranhão e Bahia. Mestranda em Saúde e Ambiente pela Universidade Tiradentes - UNIT.
} 
essa arquitetura de escolhas deve ser pensada em um âmbito de se facilitar que os indivíduos e a sociedade atinjam seus objetivos em termos de bem-estar. Compreendendo as importantes ferramentas, como as políticas públicas de saúde podem lançar mão das mesmas nas suas práticas? Assim esse artigo tem como objetivo explanar sobre a relevância da APS e a utilização das atividades educativas como estratégia de prática sustentável e subsidiária para o fortalecimento da autonomia e melhora da qualidade de vida dos indivíduos e comunidades, ressaltando o papel do profissional enfermeiro na equipe de Saúde da Família e o uso de nudge como impulsionador dos resultados.

Palavras-Chave: Atenção Primária à Saúde; educação em saúde; enfermeiro; nudge; paternalismo libertário.

\begin{abstract}
Primary Health Care (PHC) is responsible for health actions, both individually and collectively, covering health promotion, health protection, disease prevention, diagnosis, treatment, rehabilitation, harm reduction and health maintenance, in order to provide integral attention to the users of the Unified Health System, with the purpose of having a striking effect on the health conditions of the population, causing influence on the autonomy of the people and on the determinants of the health process of the population. The use of health education by the Family Health Teams (eSF) has been of great value, since it is a field with several peculiarities that contributes to the development of the individual that is based on the conception of education, which makes possible the stimulation of reflection, develops critical awareness, exercises autonomy, offers the possibility of transforming his reality and writing his own history. In public health policies we can consider the use of Nudge to drive individual and collective decision making for healthy and sustainable practices. In the case of "libertarian paternalism," this architecture of choices must be thought of in terms of enabling individuals and society to achieve their goals in terms of well-being. Understanding the important tools, how can public health policies put them in practice? Thus, this article aims to explain the relevance of PHC and the use of educational activities as a sustainable and subsidiary practice strategy to strengthen autonomy and improve the quality of life of individuals and communities, highlighting the role of the nurse practitioner in the team. Family Health and the use of nudge as a driver of results.
\end{abstract}

Key Words: Primary Health Care; health education; nurse; nudge; libertarian paternalism.

\title{
1 INTRODUÇÃO
}

Nos últimos anos pesquisas realizadas no Brasil, evidenciam a participação da Atenção Primária a Saúde (APS) na construção e consolidação do Sistema Único de Saúde (SUS), que atua desde a redução de custo, ampliação da satisfação do usuário até o princípio da equidade. Com isso, o governo federal lança políticas nacionais para otimizar e concretizar a atenção básica em todo território nacional (BRASIL, 2007). 
A APS é responsável por ações de saúde, no âmbito individual e coletivo, que abarca promoção, proteção da saúde, prevenção de agravos, estabelecimento de diagnóstico, tratamento, reabilitação, redução de danos e a manutenção da saúde, a fim de proporcionar uma atenção integral ao usuário do Sistema Único de Saúde, com a finalidade de surtir um efeito impactante nas condições de saúde da população, causando influencia na autonomia das pessoas e nos fatores determinantes do processo saúde doença da população (BRASIL, 2017).

Ao longo da história o desenvolvimento e consolidação do SUS resultaram na criação da Política Nacional da Atenção Básica (PNAB) em 2006, oficializando a Estratégia de Saúde da Família (ESF) como prioritária para desenvolver ações multidisciplinares planejadas a partir das necessidades locais, utilizando o diagnóstico de saúde do território, proporcionando efetividade, uma vez que estabelece vínculo entre os profissionais e o indivíduo/comunidade (SILVA, CASSOTI, CHAVES, 2010).

A ESF possui por objetivo reorganizar a atenção básica no Brasil, obedecendo ao que é preconizado pelo Sistema Único de Saúde. O Ministério da Saúde, assim como os gestores estaduais e municipais consideram-na como estratégia de expansão, qualificação e consolidação da atenção básica, haja vista que promove reorientação do processo de trabalho (BRASIL, 2017).

A utilização de práticas que visam promoção da saúde e a prevenção de doenças em saúde deve ser norteada por atividades educativas, que permitam o fortalecimento da atenção primária, provocando um impacto na situação de saúde da população adstrita, considerando que o enfermeiro ocupa posição de caráter decisivo e proativo na identificação das necessidades de cuidados da população, através de ações que contemplam promoção e proteção à saúde (BACKES et al., 2012).

O emprego da educação em saúde tem sido de bastante valia, por ser um campo com várias peculiaridades que contribui para o desenvolvimento do indivíduo que tem como base a concepção de educação o que possibilita estímulo da reflexão, desenvolve consciência crítica, exerce autonomia, oferece possibilidade de transformar sua realidade e escrever a própria história (FIGUEIREDO, RODRIGUES, LEITE, 2012).

Dessa forma, educação em saúde representa o empenho da equipe de saúde que busca reconhecer a realidade da população e dar a devida atenção às suas necessidades sociais em saúde de acordo com sua área de abrangência. Esta prática contribui para a autonomia das pessoas no seu cuidado, desde que estabeleça troca de conhecimentos e se configure como ato de criar e transformar a realidade, sendo importante referência 
para possibilitar as mudanças das condições de vida e de saúde (OLIVEIRA et al., 2013).

Em países cujo sistema de saúde utilizam a APS como porta de entrada, obtiveram resultados satisfatórios, haja vista que este nível de atenção subsidia a elaboração e execução de ações preventivas que objetivam a redução de internações, consultas no nível secundário, terciário e realização de exames de alto custo, reduzindo despesas, promovendo a satisfação dos clientes e acima de tudo possibilitando maior equidade da assistência à saúde (MENDONÇA, 2009).

A Enfermagem tem na ação educativa, um de seus principais eixos norteadores que se concretiza nos vários espaços de realização das práticas de Enfermagem em geral e especialmente no campo da Saúde Pública. Isso implica pensar a ação educativa como eixo fundamental para a nossa formação profissional no que se refere ao cuidado de Enfermagem em Saúde Pública e a necessidade de identificar ambientes pedagógicos capazes de potencializar essa prática (ACIOLI, 2008).

Um dos pressupostos encontrados tanto na formulação de políticas públicas, quanto no pensamento econômico atual, é o de indivíduo racional, aspecto no qual se espera um comportamento estratégico individual em meios para se atingir resultados esperados. O aspecto do indivíduo racional encontra-se ligado à formulação das políticas públicas, assim como os resultados prováveis, tendo em vista o comportamento esperado da população de acordo com aquela política (RAMIRO, FERNANDEZ, 2017).

Nas políticas públicas de saúde podemos considerar o uso do Nudge para o impulsionamento de tomada de decisões individuais e coletivas para práticas saudáveis e sustentáveis. O tema é abordado por Thaler e Sunstein (2008), onde os autores analisam os aspectos da tomada de decisão, mas também situações no cotidiano onde haveria a necessidade de "empurrões" ou "cutucadas" (nudges) para determinadas escolhas atingirem um bem-estar, seja em caráter privado ou mesmo público. Diante disso, abordam áreas como a educação, saúde, meio-ambiente e dinheiro, citando também a questão do "paternalismo libertário".

No caso do "paternalismo libertário", essa arquitetura de escolhas deve ser pensada em um âmbito de se facilitar que os indivíduos e a sociedade atinjam seus objetivos em termos de bem-estar, se valendo, inclusive, dessas orientações em forma de nudges, mas também através de informações mais claras e que correspondam à 
capacidade de racionalidade limitada entendida pelos autores (RAMIRO, FERNANDEZ, 2017)

Compreendendo as importantes ferramentas, as políticas públicas de saúde podem lançar mão do paternalismo libertário afim de obter as metas propostas e desejáveis, muitas vezes não atingidas, agregando nudge e psicologia comportamental nas suas práticas? Assim esse artigo tem como objetivo explanar sobre a relevância da APS e a utilização das atividades educativas como estratégia de prática sustentável e subsidiária para o fortalecimento da autonomia e melhora da qualidade de vida dos indivíduos e comunidades, ressaltando o papel do profissional enfermeiro na equipe de Saúde da Família e o uso de nudge como impulsionador dos resultados.

\section{ATENÇÃo PRIMÁRIA À SAÚdE E ESTRATÉGIA SAÚdE DA FAMÍLIA}

A APS ganha efetivamente relevância a partir da I Conferência Internacional sobre Cuidados Primários de Saúde, realizada pela Organização Mundial da Saúde (OMS) e o Fundo das Nações Unidas para a Infância (Unicef), que que foi realizada na cidade de Alma-Ata no Cazaquistão em 1978. O documento e acordo final, a Declaração de Alma Ata, aponta para a meta "Saúde para todos no ano 2000" e tem a APS como estratégia que norteia a adoção de ações cujo foco central são medidas preventivas e as intervenções em áreas prioritárias, como educação em saúde voltada para a prevenção e proteção, saneamento básico, nutrição, saúde da mulher e da criança, imunização, prevenção de endemias e fornecimento de medicamentos essenciais (BRASIL, 2010).

A terminologia APS normalmente é entendida como uma atenção ambulatorial especializada, oferecida por unidades de saúde que compõe um determinado sistema, de modo que estas unidades também são espaços onde ocorrem o primeiro contado dos usuários com o sistema, propiciando a estes a resolução da maior parte dos problemas por eles apresentados (LAVRAS, 2011).

A APS é responsável por realizar ações no âmbito individual e coletivo, que abrange a promoção, proteção da saúde, prevenção de agravos, estabelecimento de diagnóstico, tratamento, reabilitação, redução de danos e a manutenção da saúde, com o intuito de proporcionar atenção integral ao usuário, subsidiando um efeito impactante nas condições de saúde da população e influenciando a autonomia das pessoas com foco nos fatores determinantes do processo saúde doença da população (BRASIL, 2017). 
No Brasil, nas décadas de 70 e 80, o Movimento da Reforma Sanitária incorporava toda a ideologia discutida no mundo e traduzida pela APS, surgindo a necessidade de reorientar o modelo de saúde assistencialista e privatista vigentes na época. A iniciativa e luta das classes de trabalhadores e estudantes da área da saúde e sociedade resultaram na criação do SUS, através da Constituição Federal de 1988 e regulamentado pela Lei 8080/1990.

O nível primário de atenção à saúde destaca-se de forma significativa na construção do SUS devido sua progressão e fortalecimento nos últimos anos, em consonância com as esferas federal, estadual e municipal, academia, trabalhadores e instituições de saúde. Apresenta ainda característica harmoniosa tendo como base os sistemas de saúde (BRASIL, 2007).

Em 1991 o Ministério da Saúde dava início o Programa Nacional de Agentes Comunitários de Saúde (PACS), que teve por objetivo incluir estes profissionais nas Equipes de Saúde da Família (eSF) de modo que promovesse mudanças relevantes no método de trabalho em saúde. Para fortalecer a Atenção Básica em 1994 foi criado o Programa de Saúde da Família (PSF), hoje conhecido por ESF, iniciado em Quixadá e Sobral, dois municípios localizados no Estado do Ceará na região nordeste do Brasil (GUEDES, SANTOS, LORENZO, 2011).

Esses programas operacionalizam um modelo assistencial pautado nos princípios do SUS como forma de reorganização da produção de cuidados de saúde, objetivando a reorientação da prática assistencial em direção a uma assistência focada na família, compreendida a partir de seu ambiente físico e social (ROECKER, MARCON, 2011).

A PNAB criada no ano de 2006 e atualizada através das Portarias de $n^{\circ} 2488$ de 21 de outubro de 2011 e $\mathrm{n}^{\mathrm{o}} 2.436$ de 21 de setembro de 2017, trouxe novos elementos, corroborando a essencialidade da Política para o modelo de saúde vigente, tem por objetivo permitir o acesso universal e contínuo aos serviços de saúde promovendo o planejamento e a programação, para efetivar a integralidade em seus vários aspectos programáticos e espontâneos em meio à interdisciplinaridade, procura também desenvolver relações de vínculo e responsabilização entre equipe e usuários.

A valorização dos profissionais ocorre através da capacitação, realização, avaliação e acompanhamento sistêmico dos resultados do planejamento e programação para estimular a participação popular e o controle social (BRASIL, 2017).

A PNAB reafirma a ESF como a estratégia de consolidação do SUS e é fruto de experimentos promovidos pelos mais diversos segmentos como movimentos sociais, 
usuários do Sistema Único de Saúde, trabalhadores, gestores municipais, estaduais e federais, haja vista que estes estão envolvidos historicamente com o desenvolvimento e a consolidação do Sistema Único de Saúde.

O Ministério da Saúde, assim como os gestores estaduais e municipais consideram a ESF como estratégia de expansão, qualificação e consolidação da atenção básica, haja vista que promove reorientação do processo de trabalho com ênfase no aprofundamento dos princípios, diretrizes e fundamentos regidos pela da atenção básica, além de aumentar a resolução dos problemas promovendo impacto na situação de saúde população, propiciando ainda um excelente custo e efetividade (BRASIL, 2017).

A Estratégia é composta por uma equipe multiprofissional de médico generalista, ou especialista em Saúde da Família, ou médico de Família e Comunidade, enfermeiro generalista ou especialista em Saúde da Família, auxiliar ou técnico de enfermagem e agentes comunitários de saúde chamada de Equipe de Saúde da Família (eSF). Os profissionais de Saúde Bucal podem ser adicionados a essa equipe, bem como outros profissionais como psicólogo, nutricionista, assistente social, fisioterapeuta, e diversas especialidades médicas podem fazer parte da equipe interdisciplinar que assiste ao paciente através do Núcleo de Apoio à Saúde da Família (NASF).

A eSF atua com o propósito de assegurar a população acesso às ações de saúde integradas, voltada ao indivíduo, família, comunidade e meio ambiente e encontra-se implementada nos municípios brasileiros, priorizando ações como promoção, proteção e recuperação da saúde dos indivíduos e da família de maneira integral e contínua (DIAS et al., 2014).

Ações de vigilância em saúde devem ser realizadas pelas eSF e estas ações devem ter uma relação com o cotidiano dos indivíduos e coletividade ao que se trata de trabalho, ambiente, acolhimento humanizado, atendimento de saúde, visita no domicílio, o que permite a criação de espaço para as atividades que envolvem educação em saúde (FERNANDES, BACKS, 2010).

Segundo Escorel (2007) a ESF é tida como modelo de atenção primaria à saúde e centro ordenador das redes de atenção à saúde do SUS. Portanto faz-se necessário a adoção de técnicas voltadas às visitas domiciliares, além de realizar reuniões entre os membros da equipe multidisciplinar e a comunidade, promoção de atividades educativas com grupos determinados e execução de ações administrativas de supervisão e educação continuada.

\section{A PRÁTICA DE EDUCAÇÃO EM SAÚDE}


A Portaria $\mathrm{N}^{\mathrm{o}} 2.446$, de 11 de novembro de 2014, redefine a Política Nacional de Promoção da Saúde (PNPS) através do conceito ampliado de saúde. Propõe sua efetivação por meio do reconhecimento de valores que contemplam a produção de saúde como a solidariedade, felicidade, ética, respeito, humanização, corresponsabilidade, justiça e inclusão social (BRASIL, 2014).

De acordo com Costa (2009), o conhecimento é algo que precisa ser compartilhado entre os profissionais de saúde e a população. A educação em saúde proporciona ao cidadão e comunidade, esclarecimentos sobre prevenção de doenças e cuidados com a saúde, informando-os a respeito das transformações para organizar e reorganizar os valores.

O Ministério da Saúde define Educação em Saúde como uma metodologia educativa que organiza o conhecimento na saúde e busca a apropriação do tema pela população. É entendido ainda como um conjunto de práticas de um setor que coopera para acrescer a autonomia dos indivíduos em relação ao seu cuidado, portanto tem o dever de proporcionar o debate entre profissionais e gestores, a fim de alcançar uma atenção à saúde de acordo com suas necessidades (BRASIL, 2012). De acordo com Vasconcelos (2001) a atenção básica à saúde é essencial para o desenvolvimento das práticas educativas de saúde, devido suas particularidades.

$\mathrm{Na}$ atualidade a educação em saúde é um recurso que faz uso da comunicação buscando desenvolver habilidades nos indivíduos para que assim estes possam desenvolver seu raciocínio crítico e identificar a influência de fatores sobre sua saúde e encorajando-os a mudar os hábitos que os deixam propenso a riscos (BEZERRA et al., 2015). É considerada um processo de ação social e de experiências de aprendizagem planejadas voltadas a capacitação das pessoas para que adquiram controle sobre os determinantes da saúde, do comportamento em saúde e sobre as condições sociais que afetam seu próprio estado de saúde e dos outros (OHARA, SAITO, 2014).

Educação e Saúde são valores que não podem ser compreendidos de forma similar, como bens de consumo pessoal. Esses dois conceitos são fundamentais para o crescimento intelectual e social do indivíduo e da coletividade, tem hoje a sua concepção teórica aumentada por cientistas sociais e analistas de recursos humanos, com isso, além da conquista pessoal representa um investimento para ocorrer o desenvolvimento coletivo (MIRANDA, MALAGUTTI, 2010). 
Segundo Miranda e Malagutti (2010), a promoção da saúde está sendo descrita como o novo e promissor paradigma da saúde, onde seus principais requisitos são a ênfase na integralidade do cuidado e prevenção, com o compromisso de melhorar a qualidade de vida e estimular a participação da comunidade sendo peça fundamental para um planejamento e avaliação dos serviços.

Duarte e Almeida (2014), consideram que a ESF representou um avanço na transformação do modelo de saúde vigente, oferecendo um atendimento humanizado, isto tem melhorado a relação entre os profissionais e os usuários com criação de vínculo, fator decisivo para realização de efetivas ações de saúde, tendo a participação do enfermeiro de fundamental importância para o fortalecimento deste modelo assistencial.

\section{O ENFERMEIRO NA EDUCAÇÃO EM SAÚDE}

Por se tratar de uma ciência, a enfermagem tem como essência e especificidade o homem no seu campo individual e coletivo o que possibilita o desenvolvimento de habilidades em áreas como promoção, proteção, prevenção, recuperação a saúde, agindo como um educador e cuidador com relacionamento interpessoal de forma integral (MENDES, 2012).

No Guia Prático do Programa de Saúde da Família o enfermeiro contempla atribuições básicas como execução no nível de suas competências, ações de assistência básica, de vigilância epidemiológica e sanitária, participação do processo de programar e planejar ações, organizar o processo de trabalho no Programa de Saúde da Família, capacitar auxiliares e técnicos de enfermagem, como também os agentes comunitários de saúde, através de ações que promove qualidade de vida e controle do meio ambiente (BRASIL, 2012).

A cada dia o enfermeiro ganha espaço na área da saúde devido a pro atividade e o papel decisivo na identificação das necessidades de saúde da população o que torna o cuidado de enfermagem um componente fundamental na implementação de programas de saúde (BACKES et al., 2012).

Segundo Moura e colaboradores (2015), é notória a atuação produtiva do enfermeiro nas ações de educação em saúde que proporcionam qualidade de vida aos pacientes através da atenção primária o que demonstra a grande importância deste 
profissional como integrante da equipe multidisciplinar que compõe a ESF das Unidades Básicas de Saúde.

A promoção da saúde é aqui entendida como um dos eixos da atenção primária. Sem desconsiderar o acúmulo teórico-conceitual específico do campo da promoção, bem como a relevância dessa discussão, propõe-se, neste momento, a operacionalização da agenda da promoção a partir da APS (BRASIL, 2007).

Assim, destacando que o enfermeiro dentro do grupo multiprofissional, é um dos agentes de educação em saúde, o qual objetiva uma conexão em benefício da promoção da saúde do cliente, da família, grupos sociais e da comunidade, a sua atuação precisa ser incondicional e participativa na rotina de tarefas, devendo estar voltada para o incremento de ações de saúde e práticas de assistência (BRASIL, 2006).

\section{SUSTENTABILIDADE EM SAÚDE}

Por motivos éticos, sociais, políticos e econômicos, a enfermagem, assim como outras áreas do conhecimento, tem sido convocada a se envolver com esse importante tema para toda a sociedade, que é a sustentabilidade ambiental. Este assunto diz respeito, sobretudo, a ações que diminuam o catastrófico impacto do homem no meio ambiente, que ameaça inclusive a saúde e a vida das pessoas em todo o mundo e de como as pessoas podem ser envolvidas cada vez mais na causa (FURUKAWA, 2017).

O termo sustentabilidade passou a ser usado nas últimas décadas para designar um processo que envolve a preocupação com a preservação do meio ambiente e dos recursos naturais, mantendo-se as condições necessárias para a vida saudável do homem. A preocupação maior é como satisfazer as necessidades do homem que habita o mundo no presente sem comprometer as necessidades daqueles que habitarão o mundo no futuro (HAYDU, CAMARGO, 2013).

Esse conceito tem sido utilizado na área da saúde para se referir à promoção da saúde via educação e luta pela garantia dos direitos previstos constitucionalmente. Tem também sido utilizada como uma espécie de sinônimo para o que se entende por integralidade do tratamento, ou seja, o conjunto de ações de caráter médico e social que devem ser empreendidas para a prevenção, a melhor condução do tratamento e obtenção da cura e/ou qualidade de vida. Seu uso está sendo rapidamente assimilado por diversas instituições de saúde por se referir a práticas sustentáveis em saúde visando bem-estar geral ou qualidade de vida, a "sustentabilidade em saúde" (PICCARDI, 2014). 
Quando se refere ao termo sustentabilidade, possivelmente é associado a ações mais voltada às práticas de gestão em saúde. Já o termo humanização é muito utilizado quando se quer correlacionar o sentido à atenção direta ao paciente, à integridade do tratamento, afetividade e solidariedade. Ele é preferido entre os profissionais de saúde e é mais assimilado por voluntários, o que justifica o amplo uso do termo humanização em saúde (PICCARDI, 2014).

Estudos das áreas da Psicologia, da Educação, da Saúde, da Antropologia, da Sociologia entre outros, permitem compreender como funcionam os sistemas sociais e prever o que pode ser feito para que tenhamos condições de um padrão de vida sustentável e envolvem análises complexas e sofisticadas de processos psicológicos, sociais e culturais. Assim, a educação ambiental, a educação para saúde e a psicologia podem nos ajudar a não deixarmos para as futuras gerações a responsabilidade e as consequências de nossos comportamentos (HAYDU, CAMARGO, 2013).

\section{PATERNALISMO LIBERTÁRIO, NUDGE E PNAB}

Independentemente da classe social ou do grau de escolaridade, todas as pessoas se deparam com determinadas opções onde devem, obrigatoriamente, fazer uma escolha. As escolhas vão de acontecimentos corriqueiros como a roupa que será usada em alguma ocasião, até decisões mais complexas como comprar um imóvel, por exemplo. E é neste momento que os indivíduos podem se influenciar com os mais diversos sistemas de projeção de escolhas apresentados no nosso dia a dia.

A aplicação de técnicas de indução com a finalidade de busca por escolhas aptas a melhorar a qualidade de vida dos indivíduos deu surgimento a um movimento conhecido como Paternalismo Libertário (THALER, SUNSTEIN, 2008), que tem o nudge como um de seus principais instrumentos. Apesar de parecer contraditório, os termos podem ser compreendidos como interferência não coercitiva planejada porque, pretendendo conduzir as escolhas das pessoas, no intuito de melhorar suas vidas (paternalismo), insiste em preservar a liberdade de escolha (libertário), permitindo que as pessoas facilmente (com um custo muito baixo) possam evitar as opções apenas paternalistas.

\footnotetext{
“... é uma abordagem que preserva a liberdade de escolha, mas que autoriza tanto as instituições privadas quanto as públicas a direcionarem as pessoas em rumos que irão promover o seu bem-estar. A presença de algum tipo de paternalismo é provável sempre que tais instituições ofereçam opções que
} 
prevaleçam, a menos que as pessoas expressamente escolham não participar.” (THALER, SUNSTEIN, 2015)

Diariamente vários "empurrões" - nudge são dados para que as pessoas façam a escolha certa. Como exemplo pode ser citado o GPS, os avisos por e-mail que uma conta vai vencer, gratificação por utilizar o carro num modo mais econômico, uma mensagem de texto que avisa sobre a consulta agendada, o despertador, um plano de previdência com adesão automática e ajustes - padrão nos celulares e tablets. O governo utiliza da estratégia quando usa alertas do malefício nas embalagens de cigarros e bebidas, quando eletrodomésticos informam seu consumo de energia, quando os rótulos trazem valores nutricionais ou quando seu site é de fácil navegação, entre outros.

Qualquer nudging oficial deve ser transparente e franco, em vez de oculto e disfarçado. Aliás, a transparência deve ser inerente à prática básica, as decisões do governo, especialmente, devem ser sujeitas ao exame e crítica por parte da população. Uma vantagem importante dos nudges, em contraste com as imposições e proibições, é evitarem a coerção. Ainda assim, eles nunca devem assumir a forma de manipulação ou trapaça. O público tem de ser capaz de analisar e investigar os nudges tanto quanto quaisquer outros tipos de ações do governo (SUNSTEIN, 2015).

Um exemplo clássico do uso do paternalismo libertário na saúde é o caso da doação de órgãos muito bem abordado por Kahneman. Ele demonstra claramente os benefícios ao país com o aumento da adesão à doação ao mesmo tempo que respeita o livre arbítrio e as escolhas das pessoas (RAMIRO, FERNANDEZ, 2017):

\footnotetext{
"Um artigo publicado em 2003 observou que o índice de doação de órgãos ficou perto de $100 \%$ na Áustria, mas foi de apenas $12 \%$ na Alemanha, ficou em $86 \%$ na Suécia, mas foi de apenas $4 \%$ na Dinamarca. Essas enormes diferenças são um efeito de enquadramento, que é causado pelo formato da questão crítica. Os países com alto índice de doação têm um formulário do tipo 'optar pela exclusão', em que os indivíduos que não desejam doar devem ticar no campo apropriado. A menos que executem essa ação simples, eles são considerados doadores voluntários. Os países com baixo índice de doação não possuem formulário desse tipo: a pessoa deve ticar num campo para se tornar um doador. Isso é tudo. A melhor forma isolada de prever se a pessoa irá ou não irá doar seus órgãos é a indicação da opção default que será adotada sem ter de ticar em um campo" (KAHNEMAN, 2012, p. 466 - 467).
}

Na organização da APS no Brasil, regulamentada pela PNAB, algumas atividades implementadas pelas eSF, que é prioritariamente norteada por educação em saúde, sugerem fortemente o nudge. Percebe-se que o reforço positivo e a perspectiva de fazer parte de um grupo seleto que faz as "escolhas certas" influencia positivamente 
nas ações individuais e coletivas das pessoas, contribuindo para a sustentabilidade e eficácia dos serviços de saúde ofertados.

A visita domiciliar realizada pelo ACS a cada família é mensal e independe de solicitação da mesma. Assim, a família já aguarda em certo período do mês receber essa visita, onde conversará sobre as práticas de atividade física e alimentação adotadas, controles pressórico e glicêmico, idas à UBS, padrão de aleitamento materno, situação vacinal de crianças e adultos, adesão à tratamentos de doenças crônicas, entre outras, o que fideliza alguns comportamentos que são divididos e discutidos entre o paciente e a equipe.

Outra conduta adotada na APS é a frequência da consulta médica para pacientes com doenças crônicas como hipertensão e diabetes serem agendadas a cada três meses, se não existirem intercorrências maiores nesse período. Entre as consultas médicas o paciente faz consultas de enfermagem mensais para acompanhamento de seu tratamento e transcrição da receita médica, mantendo um controle efetivo do agravo. Essa rotina vincula o paciente a esses profissionais e ao controle da doença, despertando para práticas saudáveis no seu cotidiano, uma vez que está sendo constantemente dimensionadas pela equipe toda.

Durante a consulta de puericultura, a mãe é sempre elogiada ao relatar que utiliza as orientações acordadas entre ela e o profissional na consulta anterior, ou quando o peso da criança está adequado, quando a mesma apresenta higiene satisfatória ou com a caderneta de vacinação em dia. Assim, o plano de cuidado compartilhado é novamente elaborado e a mãe estimulada a continuar com seu belíssimo trabalho, permitindo que esse reforço positivo seja uma ótima estratégia da adesão da mãe no cuidado da criança.

A realização dos exames de rastreamento do câncer, por exemplo, segue uma frequência padrão para cada tipo de câncer. Assim as pessoas são orientadas durante as consultas e em outras oportunidades de trocas de informação, como e quando deverá realizar seus exames. Se ainda assim houver negligencia ou esquecimento, através de seus sistemas de informação, a eSF tem controle da população faltosa, assim poderá enviar através dos ACS convites para que o paciente compareça ao serviço de saúde para realizar seu exame de rotina em tempo hábil, antes que hajam manifestações da doença.

Outra atividade realizada na APS pelos membros da equipe e que corresponde a uma das ações da Vigilância em Saúde, é a "busca ativa". Nesta, o indivíduo recebe 
uma visita domiciliar sempre que, por algum motivo, deixou de comparecer a um atendimento agendado, seja para o recebimento de resultados de exames que traga um diagnóstico importante ou na vigência da necessidade de comunicação da eSF com esta família. Dessa forma, é possível por exemplo a quebra da cadeia de transmissão, no caso de doenças transmissíveis, quando se inicia rapidamente um tratamento, quando os contatos intradomiciliares são examinados, ou quando o caso é devidamente investigado e notificado.

Bem como os ACS, as visitas também são realizadas por Agentes Comunitários de Endemias (ACE). Eles observam as condições ambientais do domicílio, identificando vetores, roedores e animais peçonhentos, associando à elementos ou condições de saneamento que possam estar propiciando tais ocorrências afim de recomendar medidas afetivas para o controle dos mesmos. Receber essa intervenção também é um reforço positivo para que o quintal seja mantido limpo, melhorando os resultados da atuação dos ACE.

Outra prática adotada principalmente na formação de grupos de educação em Saúde, é de selecionar um nome para o grupo que remeta à saúde, vida, união, conquista ou vitória por exemplo e não associar o grupo a uma patologia, ou problema social. Essa estratégia melhora a adesão quando os participantes compreendem que fazem parte de algo positivo e construtivo e não de um grupo estigmatizante que só tratará de "doença" em seus encontros.

\section{CONSIDERAÇÕES FINAIS}

Aqui se propõe as práticas educativas na saúde como base para todo o processo emancipatório das comunidades, advindo do conhecimento construído nessas atividades, considerando que estas sempre devem se atentar para as experiencias prévias, sejam culturais, psicológicas ou sociais do indivíduo, de forma que ele seja um ator importante no processo.

É uma ferramenta factível e já implantada como ação inerente à APS e que ao estabelecer vínculo e confiança com a população adscrita, através das consultas, oficinas, encontros, grupos e seminários, fideliza também comportamentos mais saudáveis, boas práticas em saúde, promove ambiente mais sustentável e sobretudo eleva o indivíduo à categoria de cooperador das políticas públicas, quando se vê 
envolvido em ações positivas e que refletem hoje e futuramente na saúde e bem estar de todos e do ambiente.

Nos exemplos citados no texto sobre as atividades educativas da APS no Brasil, percebe-se a perspectiva da economia comportamental, através das visões do paternalismo libertário e do nudge que levam a novos escopos para a formulação e análise das políticas públicas vigentes. Há de se considerar que esta visão foi criada em países desenvolvidos onde se pode obter vantagens na regulação, proteção ao consumidor, questões ambientais, produção de lixo e consumo de energia e que aqui, devem ser elaboradas e pensadas estratégias que contemple as questões mais urgentes e peculiares da realidade local.

Para esse fim, se faz necessário que se aumente os estudos sobre a área da psicologia comportamental pelos países menos privilegiados, buscando atingir seus interesses, assim como ressaltar a importância de iniciativas que já vislumbram combinar desenvolvimento e sustentabilidade em saúde.

\section{REFERÊNCIAS}

ACIOLI, Sonia. A prática educativa como expressão do cuidado em Saúde Pública. Rev Bras Enferm. Brasília, 2008 jan-fev; 61(1): 117-2

BACKES, D. S. et al. O papel profissional do enfermeiro no Sistema Único de Saúde: da saúde comunitária à estratégia de saúde da família. Ciência \& Saúde Coletiva. 17(1) 223-230, 2012.

BEZERRA, I. M. P. et al. Comunicação no Processo Educativo Desenvolvido pelos Enfermeiros: As Tecnologias de Saúde em Análise. Saúde \& Transformação.

Social/Health \& Social Change, v. 5, n. 3, p. 42-48, 2015.

BRASIL. Ministério da Saúde. Pré-natal e Puerpério: atenção qualificada e humanizada - Manual técnico/ Brasília: Ministério da Saúde, 2006.

. Conselho Nacional de Secretários de Saúde. Atenção Primária e Promoção da Saúde / Conselho Nacional de Secretários de Saúde. - Brasília: CONASS, 2007.

Ministério da Saúde. Secretaria de Atenção à Saúde. Política Nacional de Humanização. Atenção Básica / Ministério da Saúde, Secretaria de Atenção à Saúde. Política Nacional de Humanização. Cadernos Humaniza SUS - Brasília: Ministério da Saúde, 2010. 256 p.: il. - v. 2.

Ministério da Saúde. Política Nacional de Atenção Básica. Portaria n 2.436 de 21 de setembro de 2017. Brasília: Ministério da Saúde, 2017 (Série E. Legislação em Saúde). 
Ministério da Saúde. Portaria no 2.446, de 11 de novembro de 2014.

Redefine a Política Nacional de Promoção da Saúde (PNPS). 2014.

COSTA, E. M. A. et al. Saúde da Família- Uma abordagem multidisciplinar. 2. ed. Rio de Janeiro: Editora Rubio, 2009.

DIAS, M. S. A. et al. intersetorialidade e estratégia Saúde da Família: tudo ou quase nada a ver?. Revista Ciência \& Saúde Coletiva, v. 19, n. 11, 2014.

DUARTE, S.; ALMEIDA, E. O papel do enfermeiro do programa saúde da família no atendimento pré-natal. Revista de Enfermagem do Centro-Oeste Mineiro, América do Norte, 0, jun. 2014. Disponível em:

<http://www.seer.ufsj.edu.br/index.php/recom/article/view/137/577>. Acesso em: 25 Ago. 2016.

ESCOREL, S. et al. O Programa de Saúde da Família e a construção de um novo modelo para a atenção básica no Brasil. Rev Panam Salud Publica, v. 21, n. 2, p. 16476, 2007.

FERNANDES M. C. P., BACKES V. M. S. Educação em saúde: perspectivas de uma equipe da estratégia da saúde da família sob a ótica de Paulo Freire. Rev Bras Enferm. Brasília, jul-ago; jul-ago; 63(4): 567-73. 2010.

FIGUEIREDO, M. F. S.; RODRIGUES NETO, J. F.; LEITE, M. T. S. Educação em saúde no contexto da Saúde da Família na perspectiva do usuário. Interface Botucatu, Botucatu. v. 16, n. 41, p. 315-329, junho 2012.

FURUKAWA, P. O. et al. Características dos profissionais de enfermagem e a prática de ações ecologicamente sustentáveis nos processos de medicação. Rev. Latino-Am. Enfermagem. 2017; 25: e 2909.

GUEDES, J. S.; SANTOS, R. M. B.; DI LORENZO, R. A. V. A Implantação do programa de Saúde da Família (PSF) no Estado de São Paulo (1995-2002). Saúde e Sociedade, São Paulo, v. 20, n. 4, p. 875-883, out./dez. 2011.

HAYDU, V. B., CAMARGO, J. C. Práticas sociais sustentáveis: Psicologia, Educação e Saúde. Departamento de Psicologia Geral e Análise do Comportamento. Universidade Estadual de Londrina, Julho de 2013. Disponível em:

http://www.uel.br/pessoal/haydu/textos/praticas-sociais-sustentaveis-psicologiaeducacao-e-saude.pdf. Acesso em: 28/11/2018.

LAVRAS, C.; Atenção primária à saúde e a organização de redes regionais de atenção à saúde no Brasil. Saúde e Sociedade, v. 20, n. 4, p. 867-874, 2011.

MENDES, K. D. S.; SILVEIRA, R. C. C.P.; GALVÃO, C. M. Revisão integrativa: Método de pesquisa para a Incorporação de evidências na saúde e na enfermagem. Texto e contexto enfermagem. vol.17 no 4, Florianópolis, Oct. /Dec. 2008. 
MENDES, L. L. M. S. Perspectivas existentes relacionadas a assistência de Enfermagem de um hospital acreditado. Revista Nursing. São Paulo, v14, n.167, p:192-196, abr. 2012.

MENDONÇA, C. S. Saúde da Família, agora mais do que nunca. Cien Saude Colet, v. 14, n. Supl 1, p. 1493-1497, 2009.

MIRANDA, S. M. R. C.; MALAGUTTI, W. Educação em Saúde. São Paulo, 2010. Editora Phorte. 312p.

MOURA, M. L. K. et al. O profissional enfermeiro como educador: um olhar para atenção primária à saúde e o NASF. Revista Interdisciplinar, v. 8, n. 1, p. 211-219, 2015.

OHARA, E. C. C.; SAITO, R. X. S. Saúde da Família: considerações teóricas e aplicabilidade. 3.ed. São Paulo, 2014.

OLIVEIRA, M. B.; CAVALCANTE, E. G. R.; OLIVEIRA, D. R.; LEITE, C. E. A.; MACHADO, M. F. A. S. Educação em saúde como prática de enfermeiros na estratégia saúde da família. Rev Rene. 2013; 14(5):894-903.

PICCARDI, T. Usos da fórmula "sustentabilidade" na área da saúde: o exemplo das práticas do voluntariado em hospitais. The Especialist, vol. 35, no 2 (170-182) 2014. ISSN 2318-7115.

RAMIRO, T.; FERNANDEZ, R. G. O nudge na prática: algumas aplicações do paternalismo libertário às políticas públicas. Periódicos UFSC. V 20, n1p1, 2017. Disponível em: https://periodicos.ufsc.br/index.php/economia/article/view/ 21758085.2017v20n1p1. Acesso em: 01/12/2018.

ROECKER, S.; MARCON, S. S. Educação em saúde: Relatos das vivências de enfermeiros com a Estratégia da Saúde Familiar. Invest. Educ. Enferm. Medellín. v. 29, n. 3, Nov. 2011.

SANTOS, Nivaldo dos; OLIVEIRA, Diego Guimarães de. A patenteabilidade de tecnologias verdes como instrumento de desenvolvimento sustentável. Revista Jurídica, [S.1.], v. 4, n. 37, p. 294-310, nov. 2015. ISSN 2316-753X. Disponível em: <http://revista.unicuritiba.edu.br/index.php/RevJur/article/view/1051/738>. Acesso em: 23 abr. 2020. doi:http://dx.doi.org/10.21902/revistajur.2316-753X.v4i37.1051.

SILVA, L. A.; CASOTTI, C. A.; CHAVES, S. C. L. A produção científica brasileira sobre a Estratégia Saúde da Família e a mudança no modelo de atenção. Ciênc. Saúde Coletiva, Rio de Janeiro, v. 18, n. cenferm UERJ. v. 18, n. 1, p. 55-60, 2010.

SUNSTEIN, Cass R. Nudging - Um guia bem breve. In: Guia de Economia Comportamental e Experimental, Org. ÁVILA, F.; BIANCHI, A. M. $1^{\mathrm{a}}$ ed. São Paulo: EconomiaComportamental.org, 2015.

SUNSTEIN, Cass S.; THALER, Richard H. O paternalismo libertário não é uma contradição em termos. Trad. Fernanda Cohen. Civilistica.com. Revista eletrônica de direito civil. Rio de Janeiro: a. 4, n. 2, 2015. Disponível em: http://civilistica.com/wp- 
content/uploads/2015/12/Sunstein-e-Thaler-trad.-Cohen-civilistica.coma.4.n.2.20151.pdf . Acesso em: 07/12/2018.

THALER, Richard H.; SUNSTEIN, Cass R Nudge: Improving decisions about Health, Wealth and Happiness. USA: Penguim Books, 2008. Disponível em: https://www.researchgate.net/file.PostFileLoader.html?id=53abe564cf57d7df1e8b45f4 \&assetKey=AS\%3A273548994646025\%401442230571326 . Acesso em: 07/12/2018.

VASCONCELOS, E. M. Redefinindo as práticas de saúde a partir da educação popular nos serviços de saúde. In: A saúde nas palavras e nos gestos: reflexões da rede de educação popular e saúde. São Paulo: HUCITEC, 2001. p.11-9. 